\title{
DISCUSSION
}

\section{Independent stress control and triaxial extension tests on sand}

\author{
READES, D. W. \& GREEN, G. E. (1976). Géotechnique 26, No. 4, 551-576.
}

\section{U. Ergun, Middle East Technical University, Ankara, Turkey}

I continued the research efforts initiated by Reades and Green on the failure criterion of cohesionless soils in the same laboratory. My concern is with the $\phi^{\prime}$ values presented by the Authors after $b=0.50$. In my opinion it is doubtful that the $\phi^{\prime}-b$ relationships in Fig. 6 represent the 'material behaviour' for the $b$ values greater than 0.50 especially for the mediumdense and loose specimens. There are two effects to consider which are not completely independent of each other:

(a) the way of straining or loading the sample;

(b) the nature of loading the platens and the possibility of interference.

In the ISC apparatus, samples can either be failed into the (constant) cell pressure, driving the axial and lateral pairs of rigid platens inwards (mode 1), or in the axial direction withdrawing the top platen (minor principal stress direction) while the lateral rigid platens are driven inwards applying the major principal stress and the constant cell pressure is the intermediate principal stress (mode 2). These two modes yield significantly different results.

The Authors and Lade and Duncan (1973) tested cuboidal samples in mode 1 and obtained similar results. Sutherland and Mesdary (1969) and Ramamurthy and Rawat (1973) employed mode 2 at high $b$ values and obtained decreasing $\phi^{\prime}$ values until extension occurred $(b=1 \cdot 0)$. It is believed that the higher resistance observed in the tests with high $b$ values in mode 1 is associated with the lower degree of freedom of the samples (due to inward moving platens) compared with mode 2 . Volume changes and rate of volume changes with respect to major principal strains indicate a more dilative behaviour in mode 1 .

The results of the ISC tests conducted on loose samples at high $b$ values in both modes and the discussion of the influence of the failure mode on the results (including those from several other testing apparatuses) can be found in Ergun (1967). Clearly, this significant effect casts serious doubt on the determination of the generalized strength behaviour of the material in the laboratory. However, the second issue is the main topic of this contribution.

The possibility of load transfer between the axial and lateral pairs of rigid platens approaching each other at the edges of the sample (i.e. platen interference) cannot be ruled out as the Authors claim. However, the platen interference is believed to have a secondary importance compared with the effect explained in the preceding paragraphs.

I believe that the platen interference exists especially in the tests with very high $b$ values (after about $b=0 \cdot 70$ ) where the two pairs of rigid platens are driven inwards at similar speeds. The Authors give certain evidence to prove that interference did not occur (p. 573). It is possible that the slightly differing gap size may not directly affect the total amount of load transfer between the platens, hence the insensitivity of $\phi^{\prime}$ to gap size may not be taken as direct evidence against interference. A photograph of a failed sample cannot be taken as sound evidence either. The observation that the stress-strain curves of a ISC test at $b=1 \cdot 0$ and a triaxial extension test are different right from the start of shearing is basically related to the effect outlined in the first section of this contribution. 

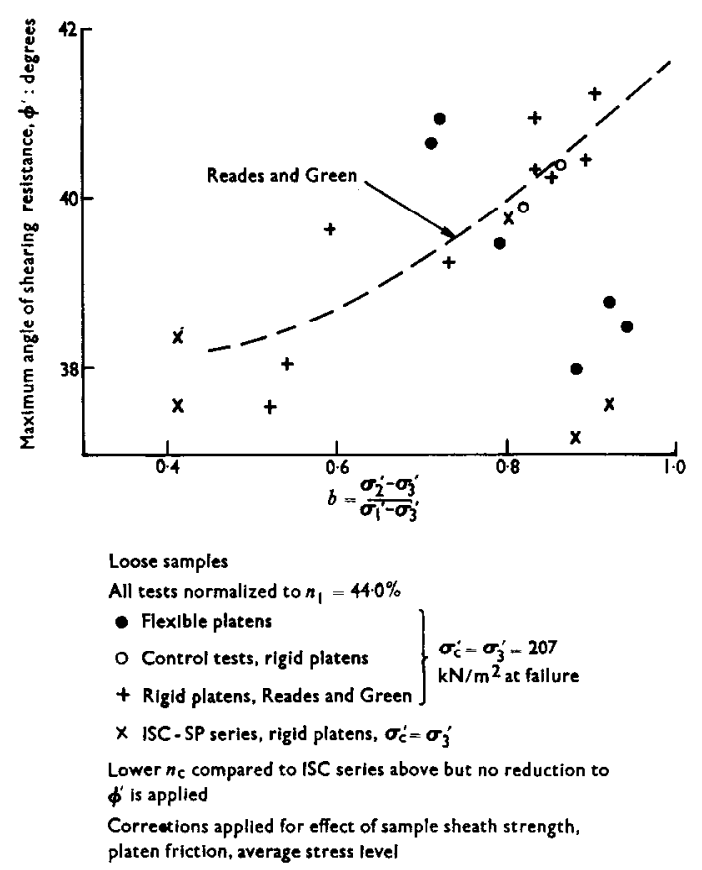

Fig. 1. Peak strengths in drained ISC tests, $b>0.4$

The observation of the similar stress-strain curves in the ISC tests near $b=1.0$ (loose samples: ISC $35,43,67,53,63)$ was interpreted by the Authors to support their argument (p. 571). Collision of the axial and lateral platens occurred in ISC 53,63, 18, ${ }^{1} 42,{ }^{1}$ and sudden increase in the axial proving ring reading could be immediately noticed. The stress-strain curves were flattening out before collision especially in ISC 18 and 42 and if these tests were stopped just before collision deciding that they had failed they would be reported as 'good tests' like ISC 35, 43, 67. It is believed that collision is not an isolated phenomenon from the pre-collision stage of an ISC test, and there must be a gradual build-up of load transfer most probably after about $60-70 \%$ of the failure load. Similarity of the stress-strain curves cannot be taken as sound evidence that interference did not occur, because this effect applies to every test performed under very similar conditions.

The following facts indicate that platen interference is very likely. (a) The ISC tests performed on the loose Ham River sand samples using flexible platens in the lateral direction (in place of stainless steel platens) show that $\phi^{\prime}$ values do not increase at high $b$ values but decrease, test conditions, corrections, etc. being the same as those in the ISC tests employing rigid platens (Fig. 1). Details can be seen in Ergun (1976). (b) Special tests ISC-SP 3, 4, 6, 7, 8 conducted on the loose Ham River sand samples at high $b$ values are also plotted in Fig. 1. These samples were first consolidated isotropically to about $840 \mathrm{kN} / \mathrm{m}^{2}$, then while the cell pressure was being lowered at a constant rate the axial rigid platens were driven to apply the major principal stress. Immediately after consolidation the oil pressure in the hydraulic jack was kept constant making use of an oil-water interface. The orientation of the principal stresses with respect to the axes of the ISC apparatus was the same as in mode 1. The only difference

\footnotetext{
${ }^{1}$ ISC 18 and 42 are not reported in the Paper. They were performed on loose samples, and the stress-strain curves can be seen in the Appendix to Reades (1972). The platens were made to collide in these tests at failure near $b=1.0$.
} 


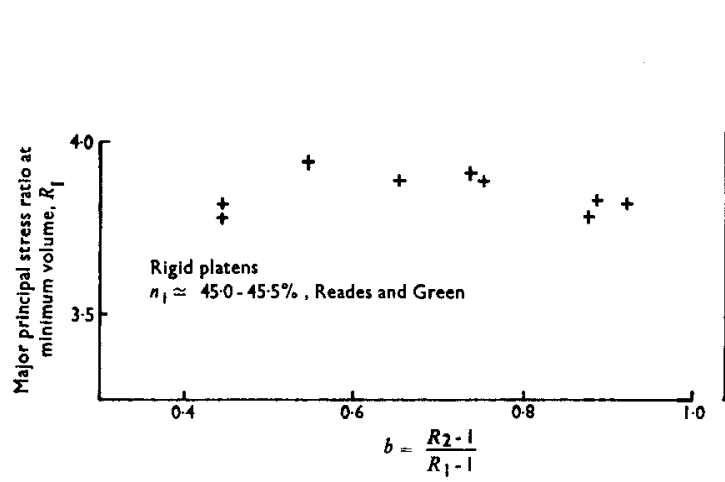

(a)

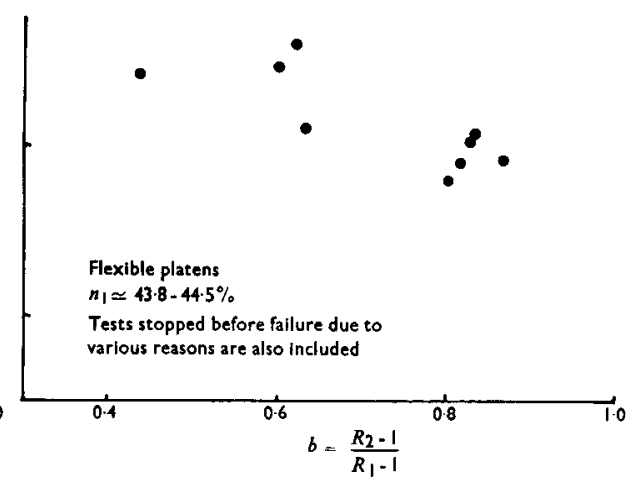

(b)

Fig. 2. Stress ratios at minimum volume in ISC tests on Ioose Ham River sand samples as measured

from mode 1 was that the belt platens were not driven in simultaneously with the axial platens. (c) An interesting observation can be made plotting the major principal stress ratio $\left(\sigma_{1}^{\prime}{ }_{1} / \sigma^{\prime}{ }_{3}\right)$ corresponding to shear at minimum volume against $b$ (Fig. 2). The main series of the ISC tests was carried out under constant cell pressure, and the major and intermediate principal stresses were increased monotonically to failure. It is certain that such a plot would not be useful under different stress paths. Strictly speaking, the three-dimensional stress paths in two ISC tests, say one at $b=0.70$ and the other $b=0.80$, are not identical but they can be practically accepted as the same. Principal strains at minimum volume are considerably smaller than those at failure whereas the $\sigma_{1}{ }_{1} / \sigma^{\prime}$ ratio at minimum volume is marginally lower than peak stress ratio, and is thus a record of a deviatoric stress without driving both pairs of rigid platens extensively. The value of $b$ is defined as $b=\left(R_{2}-1\right) /\left(R_{1}-1\right)$ in this plot where $R_{2}=\sigma_{2}^{\prime} / \sigma_{3}^{\prime}$ and $R_{1}=\sigma^{\prime}{ }_{1} / \sigma^{\prime}{ }_{3}$ at minimum volume. Comparison of the Authors' Fig. 6 and Fig. 1 and Fig. 2 is interesting.

The normal stress distribution recorded on the instrumented nested platen in the plane strain apparatus (during a test on a kaolin sample) by Sketchley (1971) indicates approximately $6 \%$ difference between the centre and the edge of the platen. This percentage could be more in a generalized test. It should be emphasized that the above considerations are not limited only to the tests in the ISC apparatus but have a general character.

\section{Authors' reply}

We wish to thank Ergun for his discussion. His additional test results using flexible platens in the intermediate principal stress direction at high $b$ values, while driving the axial and belt pairs of platens inwards and maintaining the cell pressure constant, are of particular interest. These tests are difficult to perform. Ergun's tests indicate an apparent reduction of some $2-3^{\circ}$ in the maximum angle of shearing resistance, $\phi^{\prime}$, as $b$ increases from $b=0.7$ to $b=1 \cdot 0$, so that the whole shape of the $\phi^{\prime}-b$ relationship in this range is different in Ergun's tests from ours. However, Ergun fails to provide data over the whole range of $b$ values and a proper perspective of his results is not possible. One could argue that, based on the data presented, Ergun's data fits our average line but is more scattered. It should be noted that Ergun's tests with flexible platens are of a similar type to our own (Ergun's mode 1) and not Sutherland and Mesdary type tests (Ergun's mode 2) where, while perfectly valid tests, tests at $b=1 \cdot 0$ must inevitably agree with triaxial extension tests. 
Ergun concluded that the $\phi^{\prime}-b$ relationship we obtained for $b$ greater than 0.5 did not represent real material behaviour, largely because he felt that there was a gradual build-up of stress transfer, most probably after $60-70 \%$ of the failure load. Unfortunately, neither of us has had access to Ergun's thesis and they hope that he will be able to publish his tests more fully in the near future.

We ourselves were surprised at the results of the first tests on loose samples since we had expected a constant $\phi^{\prime}$ value independent of $b$ for high porosities (see Green, 1971, Fig. 3-31). Because of this, we carried out the comprehensive test programme outlined in the paper. Based on our available test data and that of others, it seemed probable to us that significant stress transfer or platen interference was not occurring, although this was an obvious possibility. Ergun's discussion on this point is largely subjective. ${ }^{2}$ It does not appear to us that there is a significant difference between the $\phi^{\prime}$ values obtained from Ergun's special tests SP 3, 4, 6, 7 and 8 with the flexible belt platens fixed and more conventional ISC tests with both the flexible belt and the rigid axial platens closing (his Fig. 1). We are also not fully aware of the significance of Ergun's Fig. 2.

We do, however, accept the possibility that Ergun's ISC tests with flexible belt platens give a $\phi^{\prime}-b$ relationship different from ours. $\phi-b$ relationships similar to that of Ergun have recently been reported by Arthur et al. (1977) for both loose and dense samples. These were obtained using a flexible boundary plane strain apparatus (with four flexible rubber bags applying the major and minor principal stresses and two lubricated Perspex plane strain sides). Arthur et al. pointed out that it is difficult to distinguish between possible deficiencies in the many new shearing apparatuses and the various possible failure criteria. However, they concluded that a failure criterion could be developed related to equality between the rates of strain hardening and strain softening. Using this relatively simple failure criterion they were able to reconcile the different experimental $\phi^{\prime}-b$ relationships from rigid boundary and flexible boundary ISC apparatuses. They suggested that in an apparatus with two approaching rigid boundaries, strain must occur simultaneously in two directions leading to an increased strain hardening rate relative to that in a flexible boundary apparatus. According to Arthur et $a l$., therefore, our results at high $b$ values may indeed represent 'real' material behaviour.

We believe that this area of experimantal research is very complex. Further careful and well-documented experimental and theoretical work on this topic is to be encouraged.

\section{REFERENCES}

Arthur, J. R. F. Dunstan, T., Al-Ani, Q. A. I. \& Assadi, A. (1977). Plastic deformation and failure in granular media. Géotechnique 27, No. 1, 53-74.

Ergun, E. U.(1976). Study of generalized strength characteristics of granular soils in a three-dimensional apparatus. $\mathrm{PhD}$ thesis, University of London.

Green, G. E. (1971). Strength and deformation of sand measured in an independent stress control cell. Proc. Roscoe Mem. Sym., Cambridge, 285-323.

Lade, P. V. \& Duncan, J. M. (1973). Cubical triaxial tests on cohesionless soil. Jnl Soil Mech. Fdn Div. Am. Soc. Civ. Engrs. 99, SM 10, 793-812.

Ramamurthy, T. \& Rawat, P. C. (1973). Shear strength of and under general stress system. Proc. Eighth Int. Conf. Soil Mech. Fdn Engng, Moscow 1.2, 339-342.

Reades, D. W. (1972). Stress-strain characteristics of a sand under three-dimensional loading. PhD thesis, University of London.

Sketchley, C. J. (1971). Discussion, session 3. Proc. Roscoe Mem. Symp., Cambridge, 391-396.

Sutherland, H. B. \& Mesdary, M. S. (1969). The influence of the intermediate principal stress on the strength of sand. Proc. Seventh Int. Conf. Soil Mech. Fdn Engng, Mexico 1, 391-399.

\footnotetext{
${ }^{2}$ Tests 18 and 42 were not presented for the sake of simplicity; the major principal stress-strain curves for these tests lie within the band of tests presented in Fig. 15(b) with the exception of the last part of the $\left(\sigma^{\prime}{ }_{1} / \sigma^{\prime}{ }_{3}\right)$ curve for test 18 which was some $5 \%$ higher.
} 